Pacific Journal of Mathematics

ON SELF-ADJOINT DIFFERENTIAL EQUATIONS OF SECOND 


\title{
ON SELF-ADJOINT DIFFERENTIAL EQUATIONS OF SECOND ORDER
}

\author{
Ruth LiNd PotTer
}

Introduction. This paper is concerned with the behavior near $x=\infty$ of solutions of the self-adjoint differential equation

$$
\left[r(x) y^{\prime}\right]^{\prime}+p(x) y=0
$$

where $r(x)>0$ and $r(x)$ and $p(x)$ are continuous for positive values of $x$. A solution is said to oscillate near $x=\propto$ if it has no largest zero. We study the oscillation and boundedness of solutions of equations of the form (1). Repeated use is made throughout the paper of the Sturm comparison and separation theorems and of two theorems due to Leighton [6; 5]. Leighton's theorems are the following.

THEOREM $L_{1}$. If $r(x)$ and $p(x)$ are continuous and $r(x)>0$ on the interval $0<x<\infty$, and

$$
\lim _{x \rightarrow \infty} \int_{1}^{\infty} \frac{d x}{r(x)}=\propto \text { and } \lim _{x \rightarrow \infty} \int_{1}^{x} p(x) d x=\infty
$$

then every solution of ( 1 ) vanishes infinitely often on the interval $(1, \infty)$.

THEOREM $L_{2}$. If $r(x)$ and $p(x)$ are continuous, and $r(x) p(x)$ is a positive monotone function of $x$ for $x$ large, a necessary condition that solutions of (1) be os cillatory near $x=\propto$ is that not both limits

$$
\lim _{x \rightarrow \infty} \int_{1}^{x} \frac{d x}{r(x)}, \quad \lim _{x \rightarrow \infty} \int_{1}^{x} p(x) d x
$$

exist and are finite.

We proceed to the study of conditions under which solutions of equation (1)

Received May 5, 1952. The author is indebted to Profess or Walter Leight on for helpful suggestions in the preparation of this paper. Part of the work was done while the author was employed under contract N9onr-95100 with the Office of Naval Research.

Pacific J. Math. 3 (1953), 467-491 
are os cillatory.

1. Oscillation theorems. In this first section we consider the so-called "normal" form of equation (1) in which $r(x) \equiv 1$. It will be useful to set

$$
p(x)=h^{-2}(x)
$$

where $h(x)$ is positive and of class $C^{2}$ when $x>a>0$. Equation (1) then becomes

$$
y^{\prime \prime}+h^{-2}(x) y=0 \text {. }
$$

To study the oscillation of solutions of equation (1.1), it is useful to consider also the equations

$$
\begin{gathered}
{\left[h^{2}(x) z^{\prime}\right]^{\prime}+z=0,} \\
{\left[h(x) \eta^{\prime}\right]^{\prime}+\left[\frac{1}{h(x)}-\frac{h^{\prime 2}(x)}{4 h(x)}+\frac{h^{\prime \prime}(x)}{2}\right] \eta=0,} \\
{\left[h(x) \zeta^{\prime}\right]^{\prime}+\left[\frac{1}{h(x)}-\frac{h^{\prime 2}(x)}{4 h(x)}-\frac{h^{\prime \prime}(x)}{2}\right] \zeta=0 .}
\end{gathered}
$$

Nonnull solutions of these four differential equations are oscillatory ${ }^{1}$ or nonoscillatory simultaneously, for one may readily verify that the derivative of a solution of (1.1) is a solution of (1.2), equation (1.3) is obtained from (1.1) by the substitution $\eta=h^{-1 / 2}(x) y$, and (1.4) is obtained from (1.2) by the substitution $\zeta=h^{1 / 2}(x) z$.

We define

$$
H_{1}(x)=\left[\frac{1}{h(x)}-\frac{h^{\prime 2}(x)}{4 h(x)}+\frac{h^{\prime \prime}(x)}{2}\right]
$$

and

$$
H_{2}(x)=\left[\frac{1}{h(x)}-\frac{h^{\prime 2}(x)}{4 h(x)}-\frac{h^{\prime \prime}(x)}{2}\right]
$$

$1 \mathrm{~A}$ solution is said to be oscillatory on an interval if it vanishes infinitely often on the interval. 
It follows from Sturm's comparison theorem that if $H_{1}(x) \leq 0$ or $H_{2}(x) \leq 0$ for large values of $x$, the solutions of (1.1) are nonoscillatory. Similarly, it follows from Theorem $L_{1}$ that if ${ }^{2}$

$$
\lim \int_{a}^{x} \frac{d x}{h(x)}=\infty
$$

solutions of (1.1) are oscillatory if

$$
\lim \int_{a}^{x} H_{1}(x) d x=+\infty
$$

or if

$$
\lim \int_{a}^{x} H_{2}(x) d x=+\infty
$$

We proceed with a proof of the following result.

THEOREM 1.1. If

$$
\lim \int_{a}^{x} H_{2}(x) d x=+\infty
$$

the solutions of (1.1) are oscillatory.

Note first that $\lim \int_{a}^{x} h^{-1}(x) d x$ cannot be finite, for then $h^{\prime}(x) \longrightarrow-\infty$; and $h(x)$ could not be positive, as assumed. An application of Theorem $L_{1}$ completes the proof of the theorem.

The following lemma will be useful in the sequel.

LEMMA 1.2. If $h(x)$ is a positive monotone function, a necessary condition that solutions of (1.1) be oscillatory is that

$$
\lim \int_{a}^{x} \frac{d x}{h(x)}=\infty
$$

To prove the lemma let us suppose that its conclusion is false; that is, suppose

$$
\lim \int_{a}^{x} \frac{d x}{h(x)}<\infty
$$

2 All limits taken in this paper will be limits as $x \longrightarrow \infty$. Unless otherwise indicated, $a$ is a suitably chosen positive number. 
Then by a well-known theorem on infinite integrals, $\lim x h^{-1}(x)=0$, so that for any fixed value of $n, h^{-1}(x)<(n x)^{-1}$, for $x$ sufficiently large. Since solutions of the equation

$$
y^{\prime \prime}+(n x)^{-2} y=0
$$

are nonoscillatory whenever $n \geq 2$, an application of Sturm's comparison theorem yields the contradiction, and the truth of the lemma is established.

THEOREM 1.2. If

$$
\lim \int_{a}^{x} H_{1}(x) d x=\infty,
$$

a necessary and sufficient condition that the solutions of (1.1) be oscillatory is that

$$
\lim \int_{a}^{x} \frac{d x}{h(x)}=\infty
$$

The sufficiency of the condition follows from Theorem $L_{1}$ applied to equation (1.3).

To prove the necessity, let us suppose that

$$
\lim \int_{a}^{x} \frac{d x}{h(x)}<\infty
$$

Since

$$
\lim \int_{a}^{x} H_{1}(x) d x=\infty,
$$

it is readily seen that $\lim h^{\prime}(x)=+\infty$, and hence $h(x)$ is monotone for large values of $x$. It follows from Lemma 1.2 that then the solutions of $(1.1)$ are nonoscillatory, contrary to the hypothesis.

The proof of the theorem is complete.

THEOREM 1.3. If $\lim \int_{a}^{x} h^{-1}(x) d x=\infty$ and, for large values of $x$,

$$
\left[h^{\prime}(x)\right]^{2} \leq k^{2}<4,
$$

solutions of (1.1) are oscillatory. 
Under the hypotheses of the theorem,

$$
\lim \int_{a}^{x} H_{1}(x) d x \geq \lim \left[\frac{1}{2} h^{\prime}(x)-\frac{1}{2} h^{\prime}(a)+\int_{a}^{x}\left(1-\frac{1}{4 k^{2}}\right) \frac{d x}{h(x)}\right]=\infty,
$$

so that Theorem $L_{1}$ implies that solutions of (1.3), and hence that solutions of (1.1), are oscillatory.

THEOREM 1.4. If $h(x) H_{2}(x)$ is a positive monotone function, a necessary condition that solutions of $(1.1)$ be oscillatory is that

$$
\lim _{a} \int_{a}^{x} \frac{d x}{h(x)}=+\infty
$$

To prove the theorem note first that it follows from Theorem $L_{2}$ that not both the limits

$$
\lim \int_{a}^{x} \frac{d x}{h(x)}, \quad \lim \int_{a}^{x} H_{2}(x) d x
$$

can be finite. Suppose the conclusion of the theorem were false. Then the positiveness of $H_{2}(x)$ would imply that the second limit above would also be finite. From this contradiction we may infer the truth of the theorem.

The following result is useful in the application of the theory.

THEOREM 1.5. If $\lim h^{\prime}(x)=L$ exists, solutions of (1.1) are nonoscillatory if $L>2$, and oscillatory if $L<2 .{ }^{3}$

This theorem is proved by using Sturm's comparison theorem with the aid of the relation

$$
h(x)=h(a)+\int_{a}^{x} h^{\prime}(x) d x
$$

If $L=2$, solutions may or may not be oscillatory depending on $h(x)$, as the following example shows.

EXAMPLE 1.1. For the equation

$$
y^{\prime \prime}+\frac{a^{2}+1 / 4 \log ^{2} x}{x^{2} \log ^{2} x} y=0,
$$

3 Part of this theorem is contained in a theorem of Hartman and Wintner [1]. 
we have

$$
h(x)=\frac{x \log x}{\left(a^{2}+1 / 4 \log ^{2} x\right)^{1 / 2}}
$$

and $\lim h^{\prime}(x)=2$, whereas the solutions of the equation are oscillatory or not according as $a^{2}>1 / 4$ or $a^{2} \leq 1 / 4$.

COROLLARY 1.5. If $\lim \int_{a}^{x} h^{-1}(x) d x=\infty$, and $H_{1}(x)$ and $H_{2}(x)$ are nonnegative and not identically zero for large values of $x$, a necessary condition that solutions of (1.1) be nonoscillatory is $\lim h^{\prime}(x)=2$.

If

$$
\lim \int_{a}^{x} \frac{d x}{h(x)}=\infty
$$

and either

$$
\lim \int_{a}^{x} H_{1}(x) d x=\infty
$$

or

$$
\lim \int_{a}^{x} H_{2}(x) d x=\infty
$$

application of Theorem $L_{1}$ to (1.3) or (1.4), as the case may be, shows that solutions of (1.1) are oscillatory. Therefore, if solutions of (1.1) are assumed nonos cillatory,

$$
\lim \int_{a}^{x} H_{1}(x) d x<\infty
$$

and

$$
\lim \int_{a}^{x} H_{2}(x) d x<\infty
$$

in which case $\lim h^{\prime}(x)$ may be seen to exist. Since

$$
\int_{a}^{x} H_{1}(x) d x=\frac{1}{2} h^{\prime}(x)-\frac{1}{2} h^{\prime}(a)+1 / 4 \int_{a}^{x} \frac{1}{h(x)}\left[4-h^{\prime 2}(x)\right] d x,
$$




$$
\int_{a}^{x} H_{2}(x) d x=-\frac{1}{2} h^{\prime}(x)+\frac{1}{2} h^{\prime}(a)+1 / 4 \int_{a}^{x} \frac{1}{h(x)}\left[4-h^{\prime 2}(x)\right] d x,
$$

and the limit of the difference of the two integrals exists, $\lim h^{\prime}(x)$ exists. Moreover, since

$$
0<\int_{a}^{x}\left[H_{1}(x)+H_{2}(x)\right] d x
$$

$\lim h^{\prime 2}(x) \leq 4$. Therefore, by Theorem 1.5, $\lim h^{\prime}(x)=2$, and the corollary is established.

An extension of The orem 1.5 to the more general equation (1) can be made if either

$$
\lim \int_{a}^{x} \frac{d x}{r(x)}=\propto
$$

or

$$
\lim \int_{a}^{x} p(x) d x=\infty
$$

We assume that $r(x)>0$ and $p(x) \geq 0$, and that $r(x)$ and $p(x)$ are functions of class $C^{\prime}$ when $0<a<x$.

THEOREM 1.6. If

$$
\lim _{a} \int_{a}^{x} \frac{d x}{r(x)}=\infty
$$

and

$$
\lim r(x) \frac{d}{d x}[r(x) p(x)]^{-1 / 2}=L
$$

the solutions of (1) are oscillatory if $L<2$, and nonos cillatory if $L>2$.

Transforming equation (1) by the substitution

$$
t=\int_{a}^{x} \frac{d x}{r(x)}
$$

leads to the equation 


$$
\frac{d^{2} y}{d t^{2}}+r(x) p(x) y=0 \text {. }
$$

The theorem follows immediately upon application of Theorem 1.5 to this equation. (Note that $L<0$ is incompatible with the assumption $r(x) p(x) \geq 0$.)

THEOREM 1.7. If $p(x)$ is positive for large values of $x$, and

$$
\lim \int_{a}^{x} p(x) d x=\propto
$$

and

$$
\lim r(x) \frac{d}{d x}[r(x) p(x)]^{-1 / 2}=M,
$$

the solutions of (1) are oscillatory if $M>-2$ and nonoscillatory if $M<-2$.

If $y$ is a solution of equation (1), $z=r(x) y^{\prime}$ is a solution of the differential equation

$$
\left[\frac{1}{p(x)} z^{\prime}\right]^{\prime}+\frac{1}{r(x)} z=0 .
$$

Thus the solutions of (1) and those of (1.7) are oscillatory or nonoscillatory together. Application to equation (1.7) of the procedure used on equation (1) in the proof of Theorem 1.6 establishes the stated result.

The examples which follow indicate the sensitivity of the results of this section.

EXAMPLE 1.2. For

$$
y^{\prime \prime}+a^{2} x^{n} y=0,
$$

we note that

$$
h^{\prime}(x)=-\frac{n}{2 a} x^{-(n+2) / 2} .
$$

To study the equation we distinguish three cases.

Case 1: $n>-2$. Then $\lim h^{\prime}(x)=0$, so that the solutions of $(1.8)$ are 
seen to be oscillatory by Theorem 1.5.

Case 2: $n<-2$. Then $\lim h^{\prime}(x)=\infty$, and Theorem 1.5 can again be applied, showing the solutions of $(1.8)$ to be nonoscillatory here.

Case 3: $n=-2$. Then $\lim h^{\prime}(x)=1 / a$. The solutions are oscillatory if $a^{2}>1 / 4$ and nonoscillatory if $a^{2}<1 / 4$, by Theorem 1.5. Theorem 1.5 fails to give any information if $a^{2}=1 / 4 \quad\left(\lim h^{\prime}(x)=2\right)$. In this case, however, $H_{1}(x) \equiv 0$, and the solutions are nonoscillatory. The equation

$$
y^{\prime \prime}+1 / 4 x^{-2} y=0
$$

is thus in a sense a limiting equation.

EXAMPLE 1.3. For

$$
y^{\prime \prime}+\left(1 / 4 x^{-2}+e^{-x}\right) y=0
$$

since $\lim h^{\prime}(x)=2$, The orem 1.5 gives no information about the solutions. However, for large values of $x, H_{1}(x)<0$ and the solutions are accordingly nonos cillatory.

EXAMPLE 1:4. Another equation for which $\lim h^{\prime}(x)=2$ is

$$
y^{\prime \prime}+1 / 4 x^{-2} \log ^{-1} x(1+\log x) y=0
$$

The solutions of this equation are oscillatory by Theorem 1.1 since

$$
\lim \int_{a}^{x} H_{2}(x) d x=\propto
$$

The limitations of the theory of this section are indicated by the fact that from the theorems which have been given here it is not possible to determine whether the solutions of the equation in Example 1.1 are oscillatory or not.

2. Counting the zeros of a solution. We consider first the differential equation (1.1), where $h(x)>0$ and of class $C^{\prime}$ on the interval $0<x<\infty$. Let $N(a, x)$ represent the number of zeros of a solution $y(x)$ of $(1.1)$ on the interval $^{4}(a, x)$ where $a>0$. This number differs by at most one for all solutions, and hence for the present purpose can be considered as depending only

4In designating intervals it will be convenient to use the following conventions: $[a, b]$ means the interval $a \leq x \leq b,(a, b]$ means the interval $a<x \leq b,[a, b)$ means the interval $a \leq x<b,(a, b)$ means the interval $a<x<b$. 
on the differential equation and not on a particular solution.

In the preceding section it was shown that the solutions of equation (1.1) are oscillatory whenever $\lim h^{\prime}(x)<2$, and are nonoscillatory whenever $\lim h^{\prime}(x)>2$. There are equations with oscillatory solutions and others with nonoscillatory solutions for which $\lim h^{\prime}(x)=2$. Wiman [8] has given an asymptotic formula for $N(a, x)$ when $\lim h^{\prime}(x)=0$ :

$$
N(a, x) \sim \frac{1}{\pi} \int_{a}^{x} \frac{d x}{h(x)}
$$

An asymptotic formula is readily found whenever $0<\lim h^{\prime}(x)<2$, by considering the set of differential equations

$$
y^{\prime \prime}+\left(m^{2}+1 / 4\right) x^{-2} y=0,
$$

where $m$ is any real number. For a particular value of $m, N(a, x)=\frac{m}{\pi} \log x+k$ ( $k$ is a constant $)$, and $h^{\prime}(x)=\left(m^{2}+1 / 4\right)^{-1 / 2}$.

THEOREM 2.1. If, in equation $(1.1), \lim h^{\prime}(x)=m<2$,

$$
N(a, x) \sim \frac{1}{\pi}\left(\frac{1}{m^{2}}-\frac{1}{4}\right)^{1 / 2} \log x .
$$

Any differential equation included in Theorem 2.1 is also included in the stronger Theorem 2.3 given below.

The Wiman formula can be extended to an equation of the form (1).

THE OREM 2.2. If

$$
\lim \int_{a}^{x} \frac{d x}{r(x)}=\infty \text { or } \lim \int_{a}^{x} p(x) d x=\infty,
$$

then whenever

$$
\lim r(x) \frac{d}{d x}[r(x) p(x)]^{-1 / 2}=0
$$

the relation

$$
N(a, x) \sim \frac{1}{\pi} \int_{a}^{x} \sqrt{p(x) r^{-1}(x)} d x
$$

holds. 
If $\lim \int_{a}^{x} r^{-1}(x) d x=\infty$, we apply to (1) the transformation

$$
t=\int_{a}^{x} r^{-1}(x) d x
$$

and obtain

$$
\frac{d^{2} y}{d t^{2}}+r(x) p(x) y=0
$$

According to the Wiman theorem, the number of zeros $N\left(a_{1}, t\right)$ of a solution $y(t)$ of $(2.2)$ is asymptotically equal to

$$
\frac{1}{\pi} \int_{a_{1}}^{t}[r(x) p(x)]^{1 / 2} d t
$$

provided

$$
\lim \frac{d}{d t}[r(x) p(x)]^{-1 / 2}=0 .
$$

But this is equivalent, under the transformation, to the first half of the theorem. If

$$
\lim \int_{a}^{x} p(x) d x=\infty
$$

we apply the transformation

$$
s=\int_{a}^{x} p(x) d x
$$

to equation (1.7), noting that the zeros of a solution of (1) and those of a solution of (1.7) separate each other, and proceed as above.

An application of a variant of the foregoing method yields a generalization of the Wiman theorem for equation (1.1).

THEOREM 2.3. If the function $g(x)=\left[x^{2} h^{-2}(x)-1 / 4\right]^{1 / 2}$ is real and positive, and

$$
\lim x\left[g^{-1}(x)\right]^{\prime}=0,
$$


then

$$
N(a, x) \sim \frac{1}{\pi} \int_{a}^{x} \frac{g(x)}{x} d x
$$

To prove the theorem we transform $(1.1)$ by the substitution $y=x^{1 / 2} z$ and obtain

$$
\left[x z^{\prime}\right]^{\prime}+g^{2}(x) x^{-1} z=0 \text {. }
$$

The proof of the theorem may now be completed by applying The orem 2.2 to equation (2.4).

Theorem 2.3 is more general than the Wiman theorem. Applying the law of the mean to $h^{-1}(x)$, we see that the Wiman condition, $\lim h^{\prime}(x)=0$, implies $\lim x h^{-1}(x)=\propto$, and it is readily verified that whenever the Wiman condition is satisfied equation (2.3) holds. On the other hand, Theorem 2.3 applies to differential equations for which the Wiman theorem is not available; e.g., 5

$$
y^{\prime \prime}+\frac{1+\log x}{4 x^{2} \log x} y=0
$$

It should be observed that Theorem 2.3 includes all equations covered by Theorem 2.1, whereas Theorem 2.1 is not applicable to equation (2.5) since $\lim h^{\prime}(x)=2$.

Still more refined results are obtainable if instead of using the transformation which led to equation (2.4), we use the substitution $y=q^{1 / 2}(x) z$, where $q(x)$ is so chosen that $\int_{a}^{x} q^{-1}(x) d x$ diverges more slowly than $\log x$. This suggests the use of the sequence

$$
x \log x, x \log x \log _{2} x, \cdots, x \log x \cdots \log _{n} x, \ldots
$$

(cf. [6]).

To show that such a sequence can be used, the following theorem is included.

THEО REM 2.4. In the differential equation

$$
\left[r_{n-1}(x) y^{\prime}\right]^{\prime}+p(x) y=0
$$

\footnotetext{
${ }^{5}$ See Example 1.4. This equation was shown to have oscillatory solutions.
} 
where

$$
r_{0}(x)=x, \quad r_{n}(x)=r_{n-1}(x) \log _{n} x,
$$

if

$$
\lim r_{n-1}(x) \frac{d}{d x}\left[r_{n-1}(x) p(x)\right]^{-1 / 2}=0
$$

and

$$
p^{-1}(x)=o\left[r_{n-1}(x) \log _{n}^{2} x\right]
$$

then

$$
\lim r_{n}(x) \frac{d}{d x}\left[r_{n}(x) p_{1}(x)\right]^{-1 / 2}=0,
$$

where

$$
p_{1}(x)=\log _{n}^{1 / 2} x\left\{\left[r_{n-1}(x)\left(\log _{n}^{1 / 2} x\right)^{\prime}\right]^{\prime}+p(x) \log _{n}^{1 / 2} x\right\}
$$

Moreover, (2.9) does not imply (2.7).

The proof is clear once the limits in question are evaluated.

3. Boundedness of the solutions of a particular equation. In this section we study the question of boundedness near $x=+\infty$ of solutions of the self-adjoint differential equation

$$
\left[r(x) y^{\prime}\right]^{\prime}-p(x) y=0 .
$$

We assume that $r(x)$ and $p(x)$ are positive continuous functions of $x$ for $x$ large, and that $r^{\prime}(x)$ is continuous.

A CANONICAL FORM. It is useful to develop a canonical form for the solutions of (3.1). This form is suggested by the special case

$$
r(x) p(x)=k^{4} .
$$

In this instance the general solution of (3.1) may be written

$$
c_{1} e^{v(x)}+c_{2} e^{-v(x)},
$$


where $v(x)=k^{-2} \int_{a}^{x} p(x) d x$, and $c_{1}$ and $c_{2}$ are arbitrary constants.

Direct computation and an application of the fundamental existence theorem for systems of differential equations yield the following result.

THEOREM 3.1. The general solution of (3.1) may be written

$$
c_{1} u(x) e^{v(x)}+c_{2} u(x) e^{-v(x)},
$$

where $u(x)$ and $v(x)$ are functions of class $C^{2}$ which satisfy the pair of equations

$$
\begin{gathered}
r u^{3}\left[\left(r u^{\prime}\right)^{\prime}-p u\right]=-1, \\
r u^{2} v^{\prime}=1 .
\end{gathered}
$$

Since $u(x)$ is a function of class $C^{2}$ satisfying (3.2) and (3.3), $u(x)$ cannot vanish.

THEOREM 3.2. The general solution of $(3.2)$ is given by the relation

$$
u^{2}=a y_{1}^{2}+b y_{2}^{2}+2 c y_{1} y_{2}
$$

where $y_{1}$ and $y_{2}$ are linearly independent solutions of (3.1) and $a, b$, and c are any constants satisfying the relation

$$
a b=-k^{-2}+c^{2},
$$

if $k$ is the constant

$$
r(x)\left[y_{1}(x) y_{2}^{\prime}(x)-y_{1}^{\prime}(x) y_{2}(x)\right]
$$

To prove the theorem, the solution given by (3.4) can be substituted directly in (3.2).

Boundedness of Solutions of (3.2). We first prove a lemma.

LeMMA 3.3. Let $r(x), r^{\prime}(x)$, and $p(x)$ be continuous and $r(x) p(x)$ be positive and monotone for large values of $x$. If $u(x)$ is a positive solution of equation (3.2), the relations $\varlimsup u(x)=\infty$ and $\underline{\lim } u(x)<\infty$ cannot hold simultaneously.

Suppose that the hypotheses of the theorem are satisfied when $x>a$, and 
that $\left\lceil\lim u(x)=\infty\right.$ and $\underline{\lim } u(x)<\infty$. Since $u(x)$ is of class $C^{2}$, there are an infinite number of relative maximum points and of relative minimum points of $u(x)$ on $(a, \infty)$. We rewrite the equation $(3.2)$ in the form

$$
\left(r u^{\prime}\right)^{\prime}=r^{-1} u^{-3}\left(r p u^{4}-1\right)
$$

From the hypotheses of the lemma, $\lim [r(x) p(x)]$ exists and is nonnegative. If $\lim [r(x) p(x)]>0$, there exists a relative maximum point $x_{M}$ of $u(x)$ for which

$$
\left[r\left(x_{M}\right) u^{\prime}\left(x_{M}\right)\right]^{\prime}>0 \text { and } r\left(x_{M}\right) u^{\prime}\left(x_{M}\right)=0
$$

This implies that there is a positive number $\epsilon$ such that $u^{\prime}(x)>0$ when $x_{M}<$ $x<x_{M}+\epsilon$, which is impossible. If $\lim [r(x) p(x)]=0$, from equation (3.2 $)^{\prime}$ we see there is a relative minimum point $x_{m}$ of $u(x)$ for which

$$
\left[r\left(x_{m}\right) u^{\prime}\left(x_{m}\right)\right]^{\prime}<0 \text { and } r\left(x_{m}\right) u^{\prime}\left(x_{m}\right)=0 \text {. }
$$

This implies that there is a positive number $\epsilon^{\prime}$ such that $u^{\prime}(x)<0$ when $x_{m}<$ $x<x_{m}+\epsilon^{\prime}$, which is impossible.

Thus, in any case, the assumption $\overline{\lim } u(x)=\propto$ and $\underline{\lim } u(x)<\infty$ leads to a contradiction. The truth of the lemma follows.

THEOREM 3.3. Let $r(x), r^{\prime}(x)$, and $p(x)$ be continuous, and $r(x) p(x)$ be positive and monotone increasing for large values of $x$. Then every solution $u(x)$ of equation (3.2) is bounded near $x=\propto$.

We recall that a solution $u(x)$ of equation (3.2) cannot vanish, and note that if $u(x)$ is a solution, so is $-u(x)$. Let $a$ be a positive real number such that the hypotheses of the theorem are satisfied when $x>a$. Suppose then that $u(x)>0$ and let $m(x)$ and $M(x)$ be respectively the minimum value and the maximum value of $u(x)$ on $[a, x]$. Let $x_{m}$ and $x_{M}$ be such that

$$
u\left(x_{m}\right)=m(x), u\left(x_{M}\right)=M(x) .
$$

Since

$\left[r(x) u^{\prime}(x)\right]^{2}-r(x) p(x) u^{2}(x)+r(a) p(a) u^{2}(a)$

$$
+\int_{a}^{x} u^{2}(x)[r(x) p(x)]^{\prime} d x+u^{-2}(a)=u^{-2}(x)+\left[r(a) u^{\prime}(a)\right]^{2},
$$


it follows that

$$
\begin{aligned}
& {\left[r\left(x_{m}\right) u^{\prime}\left(x_{m}\right)\right]^{2}+c_{1}^{2} \leq m^{-2}(x)+r(a) p(a) m^{2}(x)+c_{2}^{2},} \\
& {\left[r\left(x_{M}\right) u^{\prime}\left(x_{M}\right)\right]^{2}+c_{1}^{\prime 2} \geq M^{-2}(x)+r(a) p(a) M^{2}(x)+c_{2}^{\prime 2},}
\end{aligned}
$$

where $c_{1}, c_{2}, c_{1}^{\prime}, c_{2}^{\prime}$ are real constants. We identify two cases according as $\left[r(x) u^{\prime}(x)\right]^{2}$ is bounded or not.

Case 1. If $\left[r(x) u^{\prime}(x)\right]^{2}$ is bounded, it follows from inequality (3.6) that $M(x)$ is bounded, and hence that $u(x)$ is bounded.

Case 2. If $\overline{\lim }\left[r(x) u^{\prime}(x)\right]^{2}=\infty$, we assume $u(x)$ is not bounded. Then

$$
\lim M(x)=\propto \text { and } \lim u(x)=\infty
$$

by Lemma 3.3. But equation $(3.2)^{\prime}$ then implies that $\left(r u^{\prime}\right)^{\prime}$ is eventually positive or $\lim \left[r(x) u^{\prime}(x)\right]^{2}=\infty$. It follows from inequality $(3.5)$ that $\lim m^{-1}(x)=\infty$ and hence that $\lim m(x)=0$. Then by Lemma 3.3, $u(x)$ is bounded.

We give a companion result when $r(x) p(x)$ is monotone decreasing.

THEOREM 3.4. If $r(x), r^{\prime}(x)$, and $p(x)$ are continuous, and $r(x) p(x)$ is positive and monotone decreasing for large values of $x$, every solution $u(x)$ of (3.2) is bounded away from zero.

The proof of this theorem is similar to that of Theorem 3.3.

The following theorem specializes Theorem 3.3 to the "normal form" of (1).

THEOREM 3.5. If $p(x)>0$ and monotone increasing for large values of $x$; then the differential equation

$$
y^{\prime \prime}-p(x) y=0
$$

is such that all solutions are monotone, and there is one solution $y_{1}(x)$ which approaches zero as $x \longrightarrow \propto$. Every solution of the differential equation (3.7) which is linearly independent of $y_{1}(x)$ is unbounded on $(0, \infty)$.

The monotone character of the solutions is apparent from the fact that if $y(x)$ is a solution of (3.7), $y^{\prime \prime}(x)$ is eventually of one sign and hence so is $y^{\prime}(x)$. The general solution can be written 


$$
y(x)=c_{1} u(x) e^{v(x)}+c_{2} u(x) e^{-v(x)},
$$

where $u(x)$ is positive, and $u(x)$ and $v(x)$ are functions of class $C^{2}$ satisfying (3.2) and (3.3). By Theorem 3.3,u(x) is bounded, and hence $\left[v^{\prime}(x)\right]^{-1}$ is bounded. Thus $v^{\prime}(x)$ is positive and bounded away from zero. This implies

$$
\varlimsup
$$

We set

$$
y_{1}(x)=u(x) e^{-v(x)}
$$

and let $y_{2}(x)$ be a positive solution linearly independent of $y_{1}(x)$. Since

$$
\lim y_{1}^{\prime}(x)=0
$$

and

$$
y_{1}(x) y_{2}^{\prime}(x)-y_{1}^{\prime}(x) y_{2}(x)=c,
$$

where $c$ is a nonzero constant,

$$
\lim y_{2}(x)=\propto,
$$

and the theorem follows.

Corollary 3.5. If $\lim \int_{a}^{x} r^{-1}(x) d x=\infty$, and $r(x) p(x)$ is a positive monotone increasing function of $x$ for $x$ large, there is one solution $y_{1}(x)$ of equation (3.1) which approaches zero as $x \longrightarrow \infty$. All solutions linearly independent of $y_{1}(x)$ approach $+\infty$ or $-\infty$ as $x \rightarrow \infty$. The solutions are all monotone.

We let $t=\int_{a}^{x} r^{-1}(x) d x$. Equation (3.1) becomes

$$
\frac{d^{2} y}{d t^{2}}-r(x) p(x) y=0,
$$

where $r$ and $p$ are to be considered functions of $t$. The theorem follows from The orem 3.5 applied to equation (3.9).

THEOREM 3.6. If $r(x), r^{\prime}(x)$, and

$$
P(x)=\frac{p(x)}{r(x)}+\frac{1}{2} \frac{r^{\prime \prime}(x)}{r(x)}-\frac{1}{4}\left[\frac{r^{\prime}(x)}{r(x)}\right]^{2}
$$


are continuous, $r(x)$ and $P(x)$ are positive, and $P(x)$ is monotone increasing for $x$ large, there is one solution $y_{1}(x)$ of equation (3.1) such that

$$
\lim r^{1 / 2}(x) y_{1}(x)=0 \text {. }
$$

For every solution $y(x)$ which is linearly independent of $y_{1}(x), r^{1 / 2}(x) y(x)$ is not bounded.

To prove this theorem we transform equation (3.1) by means of the substitution $z=r^{1 / 2}(x) y$. The resulting differential equation is

$$
z^{\prime \prime}-P(x) z=0 \text {. }
$$

Application of Theorem 3.5 to this equation yields the theorem.

EXAMPLE 3.1. For

$$
\left(x^{2} y^{\prime}\right)^{\prime}-x^{2} y=0
$$

all solutions of the corresponding equation

$$
x^{2} u^{3}\left[\left(x^{2} u^{\prime}\right)^{\prime}-x^{2} u\right]=-1
$$

are bounded since $[r(x) p(x)]^{\prime}>0$ (Theorem 3.3). The general solution of the given equation is

$$
\frac{1}{x}\left(c_{1} e^{x}+c_{2} e^{-x}\right) \text {. }
$$

EXAMPLE 3.2. For the equation

$$
\left(\frac{1}{x^{2}} y^{\prime}\right)^{\prime}-\frac{x^{2}-2}{x^{4}} y=0,
$$

$[r(x) p(x)]^{\prime}<0$. By Theorem 3.4, therefore, all solutions of

$$
\frac{1}{x^{2}} u^{3}\left[\left(\frac{1}{x^{2}} u^{\prime}\right)^{\prime}-\frac{1}{x^{4}}\left(x^{2}-2\right) u\right]=-1
$$

are bounded away from zero. Woreover, since

$$
\frac{p(x)}{r(x)}+\frac{1}{2} \frac{r^{\prime \prime}(x)}{r(x)}-\frac{r^{\prime 2}(x)}{4 r^{2}(x)}=1,
$$


by Theorem 3.6 there is a solution $y_{1}(x)$ of the given equation such that

$$
\lim x^{-1} y_{1}(x)=0
$$

and for every linearly independent solution $y(x)$,

$$
\lim x^{-1} y(x)=\infty \text {. }
$$

The general solution of this equation is

$$
c_{1} x e^{x}+c_{2} x e^{-x}
$$

EXAmPle 3.3. Consider the differential equation

$$
y^{\prime \prime}-\left(x+\frac{3}{4 x^{2}}\right) y=0 \text {. }
$$

By Theorem 3.5, one solution of this equation approaches zero, and all solutions which are linearly independent of this solution become infinite. The general solution of the equation is

$$
c_{1} x^{-1 / 2} e^{x^{2 / 2}}+c_{2} x^{-1 / 2} e^{-x^{2} / 2} \text {. }
$$

The riccati equation associated with equation (3.1). Since the solutions of equation (3.1) are nonoscillatory, the transformation $w=r(x) y \%$, applied to this equation leads to the relationship

$$
w^{\prime}=p(x)-r^{-1}(x) w^{2},
$$

which is valid for each solution $y$ of (3.1) when $x$ is sufficiently large. The differential equation (3.10) can be used to obtain additional information on the question of boundedness of solutions of (3.1).

THEOREM 3.7. If $\lim \int_{a}^{x} r^{-1}(x) d x<\infty$ and $\lim \int_{a}^{x} p(x) d x<\infty$, all solutions of (3.1) are bounded, and there is a positive constant $M$ such that

$$
\left|y^{\prime}(x)\right|<M r^{-1}(x)
$$

It is sufficient to consider only positive solutions of equation (3.1). Accordingly, we suppose $y(x)$ is any solution of (3.1) which is positive for $x$ large, and let $b$ be a positive number such that both $y(x)$ and $y^{\prime}(x)$ are of one 
sign when $x \geq b$. Equation (3.10) is then valid for such values of $x$, and $w(x)$ is of one sign. If $w(x)<0$ when $x \geq b, y(x)$ is bounded. If $w(x)>0$, when $x \geq b$ it follows from equation (3.10) that

$$
w^{\prime}(x)<p(x)
$$

and

$$
w(x)<w(b)+\int_{b}^{x} p(x) d x<K,
$$

where $K$ is a constant. Hence

$$
\frac{y^{\prime}(x)}{y(x)}<K r^{-1}(x)
$$

and

$$
\log \left|\frac{y(x)}{y(b)}\right|<K \int_{b}^{x} \frac{d x}{r(x)}<\infty
$$

Thus, $y(x)$ is bounded.

To prove the last statement of the theorem we apply the first part to the equation

$$
\left[p^{-1}(x) z^{\prime}\right]^{\prime}-r^{-1}(x) z=0
$$

for which $z=r(x) y^{\prime}$ is a solution if $y$ is a solution of (3.1).

Examples 3.1 and 3.2 show that the hypotheses of Theorem 3.7 cannot be weakened to the convergence of only one of the integrals

$$
\int_{a}^{\infty} \frac{d x}{r(x)}, \quad \quad \int_{a}^{\infty} p(x) d x
$$

4. Boundedness of nonoscillatory solutions of an equation of the form (1). In this section we study the boundedness of solutions of an equation of the form (1) when its solutions are nonoscillatory and both $r(x)$ and $p(x)$ are positive and continuous functions of $x$ for large values of $x .^{6}$ It is known that a necessary condition for the solutions of (1) to be nonoscillatory is that not both

6Sections 3 and 4 together discuss boundedness of nonoscillatory solutions whenever $p(x)$ is eventually one sign, for $p(x)$ negative and positive respectively. The case where $p(x)$ is not of one sign is not studied in this paper. 


$$
\lim _{a} \frac{d x}{r(x)}=\propto
$$

and

$$
\lim \int_{a}^{x} p(x) d x=\propto
$$

If $r(x) p(x)$ is a monotone function, the convergence of both of the aforementioned integrals is a sufficient condition that ( 1 ) have nonoscillatory solutions [5].

We state the principal theorem:

THEOREM 4.1. A necessary and sufficient condition that an equation of the form (1) with nonoscillatory solutions have all solutions bounded near $x=\infty$ is that

$$
\lim _{a} \int_{a}^{x} \frac{d x}{r(x)}<\infty
$$

Whenever the solutions of equation (1) are nonoscillatory, the transformation $w(x)=r(x) y^{\prime} / y$ leads to the Riccati equation

$$
w^{\prime}=-p(x)-\frac{w^{2}}{r(x)},
$$

which is valid for each solution $y(x)$ of $(1)$ when $x$ is sufficiently large.

Let $y(x)$ be a nonoscillatory solution of (1) such that $y(x)>0$ and $y^{\prime}(x) \neq 0$ whenever $x \geq a>0$, where $a$ has been chosen sufficiently large that $p(x)>0$ when $x \geq a$. It is sufficient to consider only solutions which are eventually positive since the negative of a solution of (1) is also a solution. Then if $x \geq a$, equation (4.1) is valid as noted above, and

$$
\frac{w^{\prime}(x)}{w^{2}(x)}<-\frac{1}{r(x)}
$$

Hence,

$$
\frac{1}{w(x)}>\int_{a}^{x} \frac{d x}{r(x)}+\frac{1}{w(a)}
$$


We assume that

$$
\lim \int_{a}^{x} \frac{d x}{r(x)}<\infty
$$

If $w(x)>0$,

$$
\frac{y^{\prime}(x)}{y(x)}<\frac{1}{r(x)}\left[\int_{a}^{x} \frac{d x}{r(x)}+\frac{1}{w(a)}\right]^{-1}
$$

by equation ( 4.2$)$, so that

$$
\log y(x)<\log \left|\int_{a}^{x} \frac{d x}{r(x)}+\frac{1}{w(a)}\right|+c,
$$

where $c$ is a constant. Accordingly, $y(x)$ is bounded. If $w(x)<0$, then $y^{\prime}(x)<0$, and $y(x)$ is bounded. Thus, whenever

$$
\lim \int_{a}^{x} \frac{d x}{r(x)}<\infty
$$

all solutions of ( 1 ) are bounded.

If $\lim \int_{a}^{x} r^{-1}(x) d x=\infty$, it follows from equations (4.1) and (4.2) that $w(x)$ is a positive, monotone decreasing function with $\lim w(x)=0$. Therefore,

$$
\lim \frac{r(x) y^{\prime}(x)}{y(x)}=0
$$

for all solutions $y(x)$ of (1). Let $y_{1}(x)$ and $y_{2}(x)$ be any two linearly independent solutions of (1) which are positive for $x$ large. From equation (4.3),

$$
\lim \frac{r(x) y_{1}^{\prime}(x)}{y_{1}(x)}=0, \quad \lim \frac{r(x) y_{2}^{\prime}(x)}{y_{2}(x)}=0 \text {. }
$$

If $c$ is the nonzero constant such that

$$
r(x)\left[y_{1}^{\prime}(x) y_{2}(x)-y_{1}(x) y_{2}{ }^{\prime}(x)\right]=c,
$$

then

$$
\frac{r(x) y_{1}^{\prime}(x)}{y_{1}(x)}-\frac{r(x) y_{2}^{\prime}(x)}{y_{2}(x)}=\frac{c}{y_{1}(x) y_{2}(x)} .
$$


The limit of the left side of the equation (4.4) is zero. Therefore, since all positive solutions of (1) are monotone increasing, at least one of $y_{1}(x)$ and $y_{2}(x)$ becomes infinite, and if

$$
\lim \int_{a}^{x} \frac{d x}{r(x)}=\infty
$$

not all of the solutions of (1) are bounded.

THEOREM 4.2. Solutions of (1) are nonoscillatory and bounded near $x=\infty$ if $r(x) p(x)$ is monotone decreasing and $\lim \int_{a}^{x} r^{-1}(x) d x<\propto$, or if $r(x) p(x)$ is monotone increasing and $\lim \int_{a}^{x} p(x) d x<\infty$.

A solution of (1) can be written in the canonical form $u(x) \sin v(x)$, where $u(x)$ and $v(x)$ are functions of class $C^{2}$ satisfying [5], the pair of equations

$$
r u^{3}\left[\left(r u^{\prime}\right)^{\prime}+p u\right]=1, r u^{2} v^{\prime}=1
$$

If $r(x) p(x)$ is monotone decreasing, there exists [5] a positive number $m$ such that $u(x)>m$. Since $\lim \int_{a}^{x} r^{-1}(x) d x<\infty$,

$$
\lim v(x)=\lim \left[v(a)+\int_{a}^{x} \frac{d x}{r(x) u^{2}(x)}\right]<v(a)+m^{-2} \lim \int_{a}^{x} \frac{d x}{r(x)}<\infty
$$

Thus, solutions of (1) are nonoscillatory. An application of Theorem 4.1 now yields the first part of the theorem.

The proof of the second half of the theorem is obtained by considering equation (1.7). If $y(x)$ is a solution of $(1), r(x) y^{\prime}(x)$ is a solution of (1.7). But by the preceding paragraph, $r(x) y^{\prime}(x)$ is nonoscillatory and bounded when $r^{-1}(x) p^{-1}(x)$ is monotone decreasing and $\lim \int_{a}^{x} p(x) d x<\infty$. Therefore, $y(x)$ is nonoscillatory. That $y(x)$ is bounded follows from a theorem of Leighton [3].

From equation (4.1) it is evident that whenever $\lim \int_{a}^{x} p(x) d x=\infty, w(x)$ is negative for every solution $y(x)$ of (1). This remark, together with the fact noted in the proof of Theorem 4.1 that, when $\lim \int_{a}^{x} r^{-1}(x) d x=\infty, w(x)$ is positive for every solution $y(x)$ of $(1)$, proves the following theorem:

THEOREM 4.3. If $\lim \int_{a}^{x} p(x) d x=\infty$, all nonoscillatory solutions $y(x)$ of (1) have the property that $y^{2}(x)$ is monotone decreasing. If $\lim \int_{a}^{x} r^{-1}(x) d x=\infty$, all nonoscillatory solutions have the property that $y^{2}(x)$ is monotone increasing.

It should be observed that the restriction to nonoscillatory solutions in 
Theorem 4.1 is not superfluous. This is illustrated by the following example.

EXAMPLE 4.1. The differential equation

$$
\left(x y^{\prime}\right)^{\prime}+\frac{1}{x} y=0
$$

has the general solution

$$
y(x)=c_{1} \sin \log |x|+c_{2} \cos \log |x| .
$$

All solutions of the equation are bounded near $x=\infty$, whereas

$$
\lim \int_{a}^{x} \frac{d x}{r(x)}=\lim \log \left|\frac{x}{a}\right|=\infty
$$

From the theorems of this section it is evident that whenever the so-called "normal form" of equation (1) with $r(x)=1$ has nonoscillatory solutions, these solutions cannot all be bounded.

5. Remarks on a theorem of Leighton. We recall that in Theorem $L_{1}$ Leighton gives, as a sufficient condition for solutions of (1) to be oscillatory, that

$$
\lim \int_{a}^{x} \frac{d x}{r(x)}=\infty, \quad \lim \int_{a}^{x} p(x) d x=\infty
$$

In the paper [6] containing this theorem there was established the existence of a sequence of tests for oscillation, each more sensitive than the preceding. This sequence was obtained by successively transforming an equation of the form (1) into an equation

$$
\left[r_{n}(x) y^{\prime}\right]^{\prime}+p_{n}(x) y=0
$$

where

$$
r_{0}(x)=x, \quad r_{n}(x)=r_{n-1}(x) \log _{n} x .
$$

It might be asked whether there is some positive function $R(x)$ with the property that whenever ( 1 ) is transformed into an equation

$$
\left[R(x) y^{\prime}\right]^{\prime}+P(x) y=0,
$$

the relations 


$$
\lim \int_{a}^{x} \frac{d x}{R(x)}=\infty, \lim \int_{a}^{x} P(x) d x=\infty
$$

would give a necessary as well as a sufficient condition for oscillation. That there is no such function is shown by the following theorem.

THEOREM 5.1. If $r(x)$ is a positive continuous function such that

$$
\lim \int_{a}^{x} r^{-1}(x) d x=\propto
$$

there exists a positive continuous function $p(x)$ such that $\lim \int_{a}^{x} p(x) d x<\propto$, and solutions of the differential equation

$$
\left[r(x) y^{\prime}\right]^{\prime}+p(x) y=0
$$

are os cillatory.

We set

$$
p(x)=\frac{1}{r(x)}\left[1+\int_{a}^{x} \frac{d x}{r(x)}\right]^{-2}
$$

The truth of the theorem then follows from Theorem 1.6.

\section{REFERENCES}

1. P. Hartman, and A. Wintner, On non-conservative linear oscillations of low frequency, Amer. J. Math. 70 (1948), 529-539.

2. E. L. Ince, Ordinary differential equations, Longmans, London, 1927.

3. W. Leighton, Bounds for the solutions of a second-order linear differential equation, Proc. Nat. Acad. Sci., U.S.A. 35 (1949), 190- 191.

4. - Principal quadratic functionals and self-adjoint second-order differential equations, Proc. Nat. Acad. Sci., U.S.A. 35 (1949), $192-193$.

5. - On self-adjoint differential equations of second order, J. London Math. Soc. 27 (1952), $37-47$.

6. differential equation, Duke Math. J. 17 (1950), 57-61.

7. C. Sturm, Sur les équations différentielles linéaires du second ordre, J. Math. Pures Appl. 1 (1836), $106-186$.

8. A. Wiman, Über die reellen Lösungen der linearen Differentialgleichungen zweiter Ordnung, Ark. Mat. Astr. Fys. 12, no. 14 (1917). 



\section{PACIFIC JOURNAL OF MATHEMATICS}

\section{EDITORS}

\section{R. U. FOEINSON}

University of California

Berkeley 4, California

E. HewitT

University of Washington

Seattle 5 , Washington
P. P. DILWOR TH

California Institute of Technology

Pasadena 4, California

E. F. BECKENBACH

University of California

Los Angeles 24, California

\section{ASSOCIATE EDITORS}

$\begin{array}{llll}\text { H. BUSEMANN } & \text { P. R. HALMOS } & \text { BØRGE JESSEN } & \text { J. J. STOKER } \\ \text { HERBERT FFDERER } & \text { IIEINZ HOPF } & \text { PAUL LÉVY } & \text { E. G. STRAUS } \\ \text { MARSHALL, IIALI } & \text { R. D. JAMES } & \text { GEORGE PÓLYA } & \text { KÖSAKU YOSIDA }\end{array}$

\section{SPONSORS}

UNIVERSITY OF BRITISH COLUMBIA

CALIFORNIA INSTITUTE OF TECHNOLOGY.

UNIVERSITY OF CAIJIFORNIA, BERKELEY

UNIVERSITY OF CAIJIFORNIA, DAVIS

UNIVERSITY OF CALIFORNIA, LOS ANGELES

UNIVERSITY OF CALIFORNI A, SANTA BARBARA

UNIVERSITY OF NEVADA

OREGON STATE COLLEGE

UNIVERSITY OF OREGON

\author{
UNIVERSITY OF SOU TIERN CALIFORNIA \\ STANFORD RESEARCH INSTITUTE \\ STANFORD UNIVERSITY \\ WASHINGTON STATE COLLEGE \\ UNIVERSITY OF WASHINGTON \\ AMERICAN MATHEMATICAL SOCIETY \\ NATIONAL BUREAU OF STANDARDS, \\ INSTITUTE FOR NUMERICAL ANALYSIS
}

Mathematical papers intended for publication in the Pacific Journal of Mathematics should be typewritten (double spaced), and the author should keep a complete copy. Manuscripts may be sent to any of the editors except Robinson, whose term expires with the completion of the present volume; they might also be sent to M.M. Schiffer, Stanford University, Stanford, California, who is succeeding Robinson. All other communications to the editors should be addressed to the managing editor, E. F. Beckenbach, at the address given above.

Authors are entitled to receive 100 free reprints of their published papers and may obtain additional copies at cost.

The Pacific Journal of Mathematics is published quarterly, in March, June, September, and December. The price per volume (4 numbers) is $\$ 8.00$; single issues, $\$ 2.50$. Special price to individual faculty members of supporting institutions and to individual members of the American Mathematical Society: $\$ 4.00$ per volume; single issues, $\$ 1.25$.

Subscriptions, orders for back numbers, and changes of address should be sent to the publishers, University of California Press, Berkeley 4, California.

Printed at Ann Arbor, Michigan. Entered as second class matter at the Post Office, Berkeley, California.

\section{UNIVERSITY OF CALIFORNIA PRESS • BERKELEY AND LOS ANGELES}




\section{Pacific Journal of Mathematics}

\section{Vol. 3, No. 2 \\ April, 1953}

William George Bade, An operational calculus for operators with spectrum

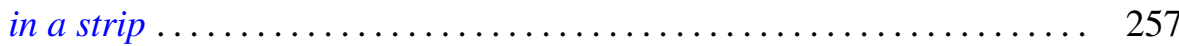

E. F. Beckenbach and Lloyd Kenneth Jackson, Subfunctions of several variables ..................................... 291

David Blackwell, Extension of a renewal theorem ................. 315

L. Carlitz, Some theorems on the Schur derivative ................ 321

Paul Arnold Clement, Generalized convexity and surfaces of negative curvature..................................... 333

Merrill M. Flood, On the Hitchcock distribution problem ............... 369

Watson Bryan Fulks, On the unique determination of solutions of the heat

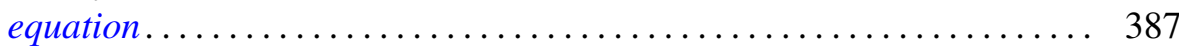

John W. Green, Length and area of a convex curve under affine transformation .................................... 393

William Gustin, An isoperimetric minimax .................. 403

Arthur Eugene Livingston, Some Hausdorff means which exhibit the Gibbs'

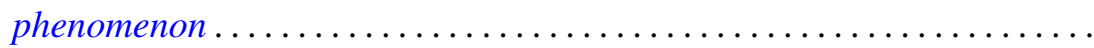

Charles Loewner, On generation of solutions of the biharmonic equation in the plane by conformal mappings ..................... 417

Gábor Szegő, Remark on the preceding paper of Charles Loewner ....... 437

Imanuel Marx and G. Piranian, Lipschitz functions of continuous

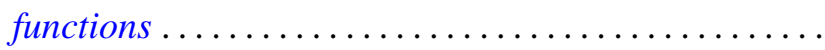

Ting-Kwan Pan, The spherical curvature of a hypersurface in Euclidean space ..

Ruth Lind Potter, On self-adjoint differential equations of second order ...

E. H. Rothe, A note on the Banach spaces of Calkin and Morrey...

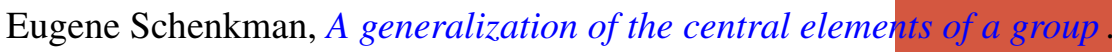

A. Seidenberg, A note on the dimension theory of rings .. . . 